\title{
Théologiques
}

\section{Superstition et divination}

\section{Guy Lapointe}

Volume 8, numéro 1, printemps 2000

La superstition

URI : https://id.erudit.org/iderudit/005013ar

DOI : https://doi.org/10.7202/005013ar

Aller au sommaire du numéro

Éditeur(s)

Faculté de théologie de l'Université de Montréal

ISSN

1188-7109 (imprimé)

1492-1413 (numérique)

Découvrir la revue

Citer ce document

Lapointe, G. (2000). Superstition et divination. Théologiques, 8(1), 5-8. https://doi.org/10.7202/005013ar d'utilisation que vous pouvez consulter en ligne.

https://apropos.erudit.org/fr/usagers/politique-dutilisation/ 


\title{
Superstition et divination
}

\author{
Guy LAPOINTE \\ Faculté de théologie \\ Université de Montréal
}

Ce que nous appelons superstition n'est-elle pas la religion des autres?

Maurice Gruau ${ }^{1}$

Superstition, divination, religion : ces trois termes constituent un entrecroisement dont on a bien du mal à saisir non seulement la dynamique interne, mais surtout la rationalité de chacune des expériences que ces mots recèlent. De ces trois termes, celui de superstition est, pour bien des personnes, carrément péjoratif et exprime un jugement de valeur, tout comme le mot secte aujourd'hui. Pour d'autres, superstition et divination font partie de ce "bricolage ", si caractéristique de tout système religieux et même culturel. A cet égard, la superstition, pas plus que la divination ne seraient vraiment pas opposées à la religion.

Autant que du bons sens dont parle Descartes, on pourrait dire de la superstition et de la divination qu'elles sont les choses du monde les mieux partagées. Nulle société, au long de l'histoire humaine, qui ne les ait, à sa façon, connues et pratiquées. On peut affirmer, sans risque de se tromper, que les pratiques de superstition et de divination font partie de nos sociétés modernes occidentales, et qu'elles demeurent, somme toute, moins marginales qu'on ne serait porté à le croire. Pour en saisir l'importance - et si les médias sont des reflets de notre vie

1. L'homme rituel. Anthropologie du rituel catholique français, Paris, Métailié, 1999. 
en société — on n'a qu'à se promener sur les réseaux d'Internet pour se rendre compte que tous les journaux du monde, des plus sérieux au plus cabotins, ont leurs sites astrologiques. Il reste néanmoins que ces pratiques, surtout en regard des rationalités scientifiques et des hautes technologies que nous connaissons, semblent avoir un statut particulier, du fait qu'elles laissent soupçonner un savoir essentiellement différent de tous les savoirs humains et, en un certain sens, infiniment supérieur.

Comment expliquer cet attrait, non pas nouveau, mais récurrent de la divination et de la superstition dans nos sociétés modernes? Peut-on parler d'un retour de l'irrationnel? Plutôt que de retour, on peut dire que des croyances, sur lesquelles on avait pieusement mis le couvercle jusque-là, semblent pouvoir s'exprimer sans honte, même chez des intellectuels et des scientifiques. Jusque dans un passé récent, du moins pour notre situation sociale, il y avait des instances officielles du savoir et de la science : l'Université, l'État, les religions instituées. Or ces trois instances ont reculé. Du coup, des croyances jugées jusque-là " parallèles » ou contraires à la religion, par exemple, s'expriment au grand jour et sont parfois mises sur le même pied que les pratiques liées à une institution religieuse. "Ça vaut bien les croyances des chrétiens ", entendons-nous. Dans ce sens, il est clair, pour ne parler que de ce que je connais mieux, que depuis toujours, le christianisme a voulu assécher la superstition, sans cependant jamais y parvenir.

Divination, superstition, religion, ces trois pratiques répondent, à leur façon, aux angoisses de tous les temps, autant aux "petites angoisses » qu'aux grandes inquiétudes ${ }^{2}$. Alors que le terme superstition, comme son étymologie latine l'indique, superstitio, d'un verbe archaïque superstitare, protéger, de super, au-dessus, et de stare, se tenir, cherche à voir au-delà, par-dessus, pour s'attirer la protection des dieux, le terme divination suppose toujours une appropriation plus ou moins poussée du savoir — et d'un savoir dont les dieux euxmêmes ou une force invisible sont garants - par un groupe restreint de spécialistes. Vouloir connaître l'avenir, se relier aux dieux, à un Dieu, lesquels, tout compte fait, nous menacent toujours. Pour

2. Voir: Jean DELUMEAU, Rassurer et protéger: le sentiment de sécurité dans l'Occident d'autrefois, Paris, Fayard, 1989. 
reprendre une citation mise en exergue de l'article de Christian StGermain : "Un dieu qui ne menacerait, ni ne rassurerait, en quoi serait-il un dieu? " N'est-il pas vrai que c'est lorsque les dieux ne nous menacent plus que la religion meurt? J'ajouterais une autre réflexion. Il m'apparaît que de tout temps, et particulièrement dans notre modernité avancée, les pratiques de superstition et de divination révèlent un aspect ludique. Cela permet de sortir d'un monde trop programmé, trop asservi à des solutions techniques, sorte d'ultime recours, ou porte de sortie.

C'est à vouloir susciter chez les lecteurs et les lectrices de Théologiques une réflexion sur quelques dimensions de la superstition et de la divination, que ce numéro a été pensé et mis en oeuvre. Dans un premier article, Jean-Claude Breton montre comment les pratiques de superstition gagneraient à recevoir, dans un contexte interreligieux, des interprétations plutôt positives qu'uniquement négatives, celles-ci considérant la superstition comme une sorte de déviance de la religion. Micheline Laliberté tisse, avec une connaissance peu commune de l'époque, les liens entre religion populaire et superstition au Moyen Age. Pour sa part, Pietro Boglioni aborde le phénomène de la divination au Moyen Age. Il le fait en montrant, ce qu'il appelle, les avatars d'une pastorale ambiguë. Il montre que l'Église du temps a eu bien du mal à contenir ces pratiques divinatoires.

La résurgence des pratiques de superstition, de magie dans notre contexte de modernité, amène Sophie Tremblay à pointer certains défis lancés à la réflexion théologique pour saisir la significaton des pratiques de magie et de superstition. Enfin Christian St-Germain, dans un article extrêmement fouillé, nous invite à surprendre la dimension poétique de l'acte divinatoire, à en chercher et à en interpréter le sens, à y reconnaître le mouvement éthique qui se joue à même les angoisses du devenir incertain. Ces pratiques de la Rome ancienne, si souvent évoquées dans l'imaginaire populaire, n'ont-elles pas, particulièrement de nos jours, laissé des traces dans le système religieux chrétien?

Le projet de ce cahier était à la fois vaste et ambitieux. Cependant, nous ne prétendons pas présenter un tableau exhaustif de ce qui se vit autour des pratiques de superstition et de divination. Nous avons tout simplement voulu en déceler quelques traces à même l'éclairage de l'histoire et de l'interprétation, espérant que la question pourra être 
reprise en nous permettant une interprétation qui tienne compte de la double dimension d'attitude mentale et d'institutions sociales de la superstition et de la divination. 